\title{
Approach to enhance trace gas determinations through multi-satellite data fusion
}

\author{
Elisabeth Weisz* and W. Paul Menzelø \\ University of Wisconsin-Madison, Space Science and Engineering Center, Madison, \\ Wisconsin, United States
}

\begin{abstract}
The imaging instruments on the polar-orbiting S-NPP and NOAA-20 satellite platforms [e.g., Visible Infrared Imaging Radiometer Suite (VIIRS)] and on geostationary GOES and Himawari platforms [e.g., Advanced Baseline Imager (ABI) and Advanced Himawari Imager (AHI)] have high horizontal spatial resolution but coarse vertical information about tropospheric gases and temperatures. An approach for fusing a high spatial resolution imager (e.g., VIIRS, ABI, and AHI) and a high information content sounder or trace gas monitor [e.g., Cross-track Infrared Sounder (CrIS) and TROPOspheric Monitoring Instrument (TROPOMI)] offers the opportunity to construct retrieval products (e.g., trace gas concentrations of $\mathrm{CO}, \mathrm{SO}_{2}$, and $\mathrm{NH}_{3}$ ) via product fusion at high spatial resolution. Furthermore, these products can also be provided at high temporal resolution when geostationary imager (ABI and $\mathrm{AHI}$ ) data are used in the product fusion. Promising results from VIIRS/CrIS, VIIRS/TROPOMI, and AHI/TROPOMI fusion are presented. (C) The Authors. Published by SPIE under a Creative Commons Attribution 4.0 Unported License. Distribution or reproduction of this work in whole or in part requires full attribution of the original publication, including its DOI. [DOI: 10.1117/1.JRS.14.044519]
\end{abstract}

Keywords: satellite remote sensing; data fusion; atmospheric trace gases; multi-sensor; infrared spectral range; high spatial resolution imager; high spectral resolution sounder.

Paper 200708 received Sep. 24, 2020; accepted for publication Nov. 19, 2020; published online Dec. 7, 2020.

\section{Introduction}

Synergistic use of high spatial resolution imager data with trace gas information provides advantages in various applications over use of individual data sets alone. For example, the high vertical resolution of hyperspectral sounding products and the high horizontal and temporal resolution of imager radiances can be blended to benefit severe weather monitoring and prediction systems. The "imager plus sounder" (or imager/sounder) data fusion method to construct high spatial resolution radiance and retrieval products has been previously described in Refs. 1 and 2. These results confirmed that the reach of either instrument can be extended through combination via the fusion approach in an efficient manner even allowing real-time applications. In this paper, we follow a similar fusion approach to infer trace gas information from sounders or monitoring sensors at imager high spatial resolution (750 $\mathrm{m}$ to $2 \mathrm{~km}$ ). This shall be referred to as imager/ trace gas fusion in this paper. Specifically, Cross-track Infrared Sounder (CrIS) coarse resolution retrievals of trace gases are mapped onto Visible Infrared Imaging Radiometer Suite (VIIRS) high spatial resolution. In addition, fusion of TROPOspheric Monitoring Instrument (TROPOMI) data with LEO (low Earth orbiting) as well as geostationary orbit (GEO) imagers to enhance monitoring of trace gas emissions from fire, volcano, and industrial sources is explored. Specific focus is put on the synergy of GEO and LEO observations [e.g., Advanced Himawari Imager (AHI) and TROPOMI] since it allows the tracking of the trace gases during high-emission events (e.g., volcanic eruption or industrial pollution) in time sequences of the fused products. These new products are shown to significantly enhance information that can be used in nowcasting, warning, and decision-making operations.

A brief description of the satellite sensors and the data fusion methodology is provided in Sec. 2. Four example applications using VIIRS/CrIS, VIIRS/TROPOMI, and AHI/TROPOMI

*Address all correspondence to Elisabeth Weisz, elisabeth.weisz@ssec.wisc.edu 
pairings are presented and discussed in Sec. 3. Section 4 summarizes the work and its implications for simulating geostationary hyperspectral soundings and future instrument design.

\section{Satellite Sensors and the Product Fusion Methodology}

\subsection{Satellites Instruments}

The instruments of interest in this paper include the following. The CrIS, ${ }^{3}$ onboard the National Oceanic and Atmospheric Administration (NOAA) operational Suomi-National Polar-orbiting Partnership (S-NPP) and NOAA-20 platforms in low Earth orbit (LEO), is a high spectral resolution (also simply referred to as hyperspectral) infrared interferometer providing very accurate infrared radiance measurements in more than 2200 channels (in full spectral resolution mode), which are used to produce high vertical resolution temperature and humidity profile retrievals. ${ }^{4}$ The CrIS field-of-view (FOV) size is $\sim 14 \mathrm{~km}$ at nadir. On the same platforms, the VIIRS ${ }^{5}$ offers high spatial $750 \mathrm{~m}$ resolution visible, near-infrared, and infrared images of ocean, land, and atmospheric features that can be co-located with the CrIS. Also in polar orbit since 2017, the TROPOMI has been part of the Sentinel-5 Precursor ${ }^{6}$ developed by the European Space Agency (ESA). Level 2 products at $7 \mathrm{~km}$ resolution include trace gas concentrations of carbon monoxide (CO), sulfur dioxide $\left(\mathrm{SO}_{2}\right)$, and ammonia $\left(\mathrm{NH}_{3}\right)$. In geostationary orbit (GEO), the Japanese Himawari and the Geostationary Operational Environmental Satellite (GOES)-R series host the $\mathrm{AHI}^{7}$ and Advanced Baseline Imager (ABI), ${ }^{8}$ respectively. These radiometers are sensitive in the visible, near-infrared, and infrared parts of the spectrum and provide radiance measurements and imagery at high 0.5 to $2 \mathrm{~km}$ spatial and hourly or better temporal resolution that are used for monitoring changes in the Earth's weather.

\subsection{Product Fusion Method}

Data from these GEO and LEO instruments are combined to generate tropospheric trace gas products with high spatial resolution that can identify small-scale features, especially changes in concentration. This can be accomplished through LEO fusion, such as VIIRS/CrIS or VIIRS/ TROPOMI, but also through GEO/LEO fusion (e.g., AHI/TROPOMI). The latter, i.e., GEO/ LEO fusion, offers not only spatial details but also hourly (or better) temporal changes in the trace gas concentrations that are important for tracking their motion through the troposphere.

To achieve sensitivity to trace gases at high spatial resolution, we use the product fusion approach detailed in Ref. 2. In general, the fusion approach is used to transfer low (coarse) spatial resolution (LORES) data products to high spatial resolution (HIRES), also referred to as spatial fusion. The main step is a nearest neighbor search — which is carried out by a multi-dimensional binary search tree (abbreviated k-d tree) ${ }^{9}$ algorithm — between the imager radiances at original high (pixel) spatial resolution (i.e., HIRES) and coarse (FOV) spatial resolution (i.e., LORES). Geographical averaging is used to obtain imager radiance data at LORES resolution, and HIRES and LORES geolocation data (i.e., the imager and the sounder latitude and longitude values) are used as additional predictors. The search finds, for each high spatial resolution pixel, the $N$ nearest neighbors-in radiance and geolocation space - of low spatial resolution FOVs. Then the products (e.g., the trace gas concentrations) at coarse spatial resolution (the trace gas instrument FOVs) associated with the $N$ nearest neighbors (i.e., best matches) found in the k-d tree search are averaged to create the new product estimate at the imager's high spatial resolution.

As mentioned above, we use the k-d tree algorithm for a nearest neighbor search on imager infrared radiances (and geolocation). For VIIRS, radiometric information consists of the split window near 10.8 and $12.0 \mu \mathrm{m}$ and for ABI and AHI a selection is made from 6.2, 7.0, $7.3,8.5,10.4,11.2,12.4$, and $13.3 \mu \mathrm{m}$ radiances that enhances sensitivity to mid- and upper tropospheric levels.

When working with a time sequence of GEO imager (such as AHI) measurements, the GEO data at the LEO overpass time (TROPOMI for example) are used to achieve the product fusion. ${ }^{2}$ It is important to note that the "LORES to HIRES" spatial fusion with the GEO data occurs only once at the LEO overpass time, denoted hereafter as $t_{0}$. Then a temporal fusion approach is used 
Step 1

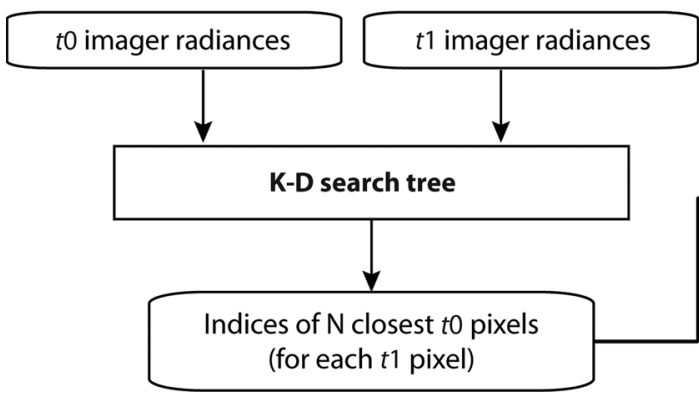

Step 2

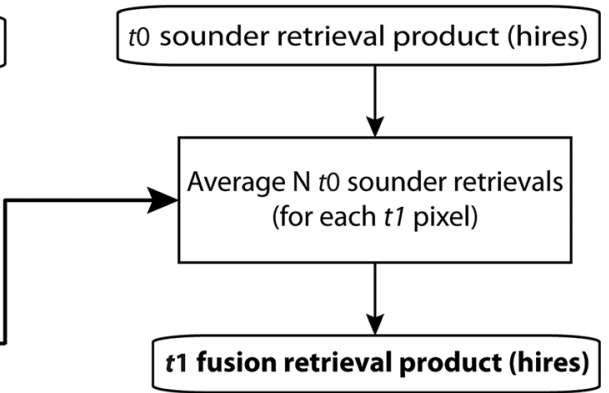

Fig. 1 Schematic of the LEO sounder to GEO imager temporal resolution fusion approach. All input and output data are at high spatial resolution (hires).

to transfer the product fusion results at full imager spatial resolution from time $t_{0}$ to previous or subsequent times $\left(t_{1}, t_{2}, t_{3}, \ldots\right)$ as illustrated in Fig. 1.

Hence, at a subsequent time $t_{1}$, the GEO radiances for a given pixel are matched with $N$ GEO pixel radiances from time $t_{0}$ through a k-d tree search and then the associated LEO products (e.g., TROPOMI trace gas concentrations) for those $N$ pixels at $t_{0}$ are averaged to produce the $t_{1}$ GEO/ LEO fusion product. The same process continues for time $t_{2}$, when each GEO pixel is connected to $\mathrm{N} t_{1}$ GEO pixels and the $t_{1}$ GEO/LEO fusion products associated with those pixels are averaged to produce the $t_{2}$ GEO/LEO fusion product. This procedure can also be applied for times previous to the overpass time. How far in time this process can be applied reliably depends on the synoptic situation, but we find that within three hours of LEO overpass time GEO/LEO fusion results are useful in most situations. ${ }^{10}$

It should be emphasized that the first step, i.e., the multi-dimensional k-d tree search, is always done on imager radiances-either between high- and low-spatial resolution imager radiances (in the case of spatial fusion) or between imager radiances from subsequent time steps (in the case of temporal fusion). In the second step, the sounder data, which is either of low spatial and/or low temporal resolution, is averaged over the suggested matches (as described above).

\section{Product Fusion Results and Discussion}

\subsection{Capturing Volcanic $\mathrm{SO}_{2}$ in More Detail with VIIRS/TROPOMI Fusion}

VIIRS and TROPOMI measurements are combined through the fusion method to delineate $\mathrm{SO}_{2}$ detection (noted in Dobson units, DU) at finer resolution. TROPOMI $7 \mathrm{~km}$ measurements covering a rather mild eruption of Mexico's Popocatépetl volcano on March 18, 2019 are fused with VIIRS $750 \mathrm{~m}$ split window radiance measurements at 10.8 (bandwidth 1.0) and 12.0 (bandwidth 1.0) $\mu \mathrm{m}$. The VIIRS split window offers sensitivity to the total column of tropospheric moisture and hence distinguishes air masses. Figure 2 shows an initial fusion result accomplished with the VIIRS split window k-d search identifying the five (i.e., $N=5$ ) TROPOMI measurements that should be averaged for each VIIRS pixel location.

The improvement in resolution is evident. Fusion is offering more detail of the $\mathrm{SO}_{2}$ distribution east of the eruption over ocean but less over land. The k-d tree search is more successful over ocean where the background is more uniform. For this modest $\mathrm{SO}_{2}$ signal, the pixel to pixel surface variations over land compromised the effectiveness of the search engine relying on the infrared split window radiances.

To better demonstrate the VIIRS/TROPOMI product fusion, another volcanic eruption showing a stronger $\mathrm{SO}_{2}$ signal in the TROPOMI measurements, ${ }^{11}$ the Krakatoa (Indonesia) eruption on December 23, 2018 is examined. Figure 3 shows the TROPOMI total vertical column $\mathrm{SO}_{2}$ as well as the VIIRS/TROPOMI fusion results. Improvement in spatial detail is apparent, especially in Fig. 3(d), which magnifies the nearby $\mathrm{SO}_{2}$ plume. 


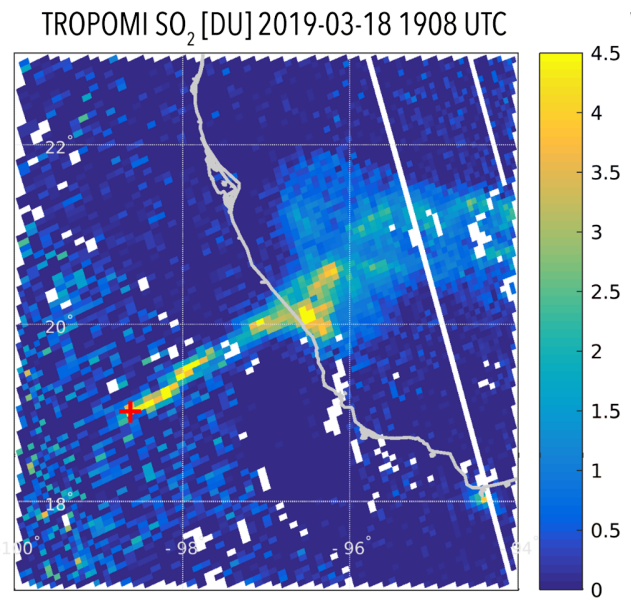

(a)

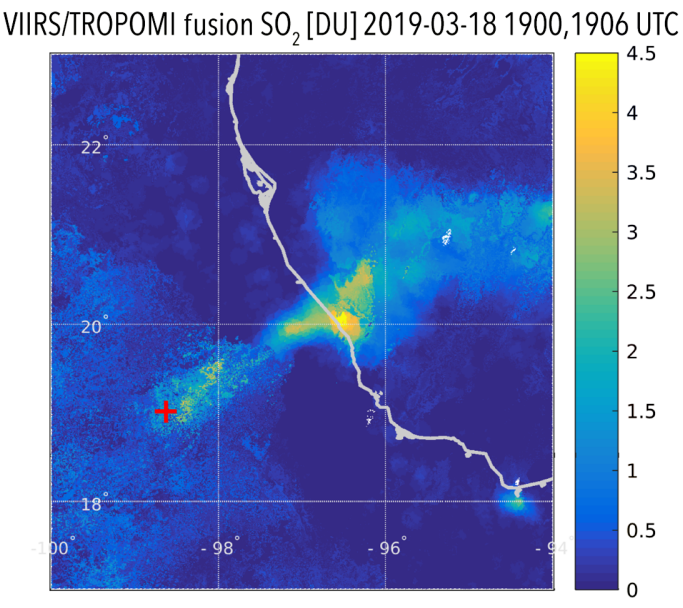

(b)

Fig. 2 (a) TROPOMI detection of $\mathrm{SO}_{2}$ in DU on March 18, 2019 and (b) VIIRS/TROPOMI fusion using a split window k-d tree search. Location of the Popocatépetl volcano (central Mexico) is marked by a red plus sign.

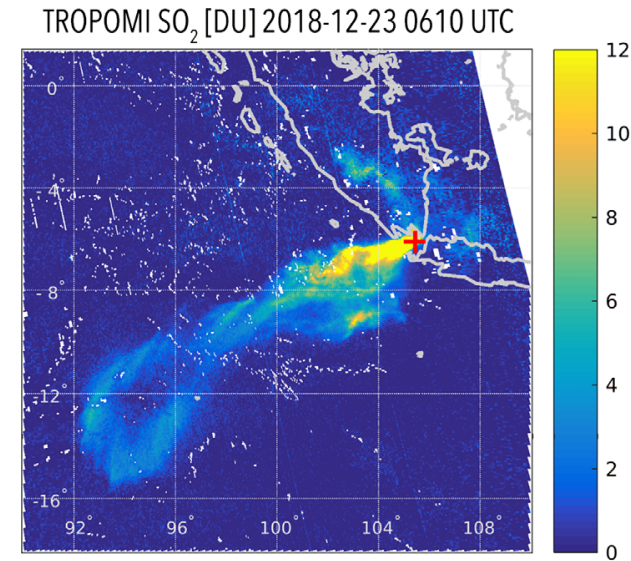

(a)

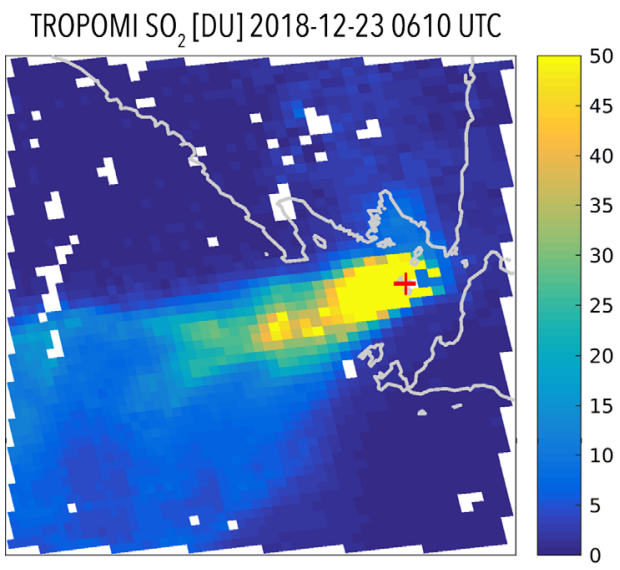

(c)

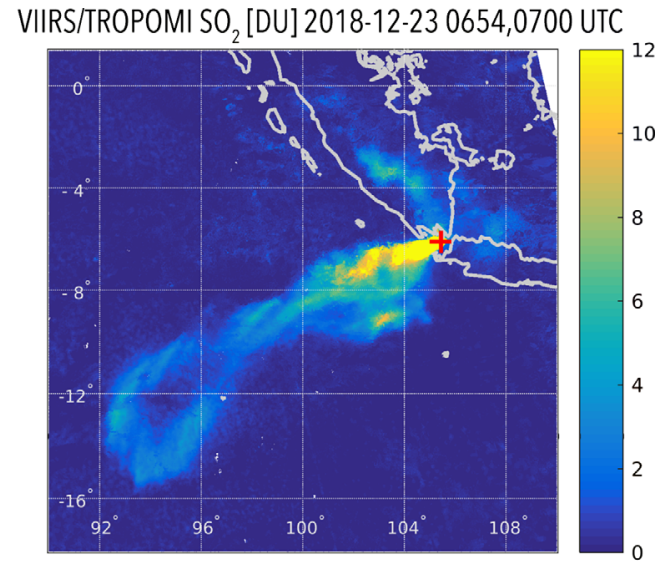

(b)

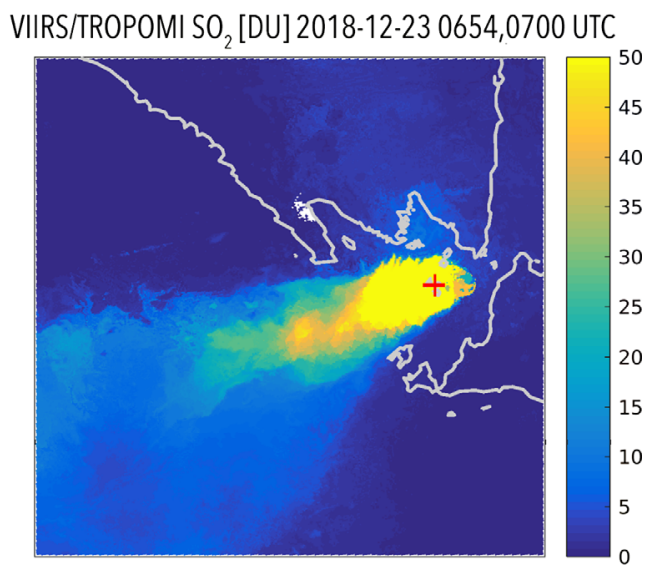

(d)

Fig. 3 Krakatoa, Indonesia, volcanic $\mathrm{SO}_{2}$ [DU] plume detected by (a), (c) TROPOMI on December 23, 2018 and (b), (d) the VIIRS/TROPOMI fusion results. (c), (d) are enlarged versions of (a) and (b) in the vicinity of the eruption center (location of Krakatoa is denoted by a red plus sign) highlighting the difference in spatial resolution between (a), (c) TROPOMI and (b), (d) the fusion product. Note $\mathrm{SO}_{2}$ range is 0-12 DU and 0-50 DU in the upper and lower panels, respectively. 
These examples of VIIRS/TROPOMI $\mathrm{SO}_{2}$ fusion offer a demonstration of the possibilities for enhanced spatial resolution (by an order of magnitude) as well as the challenges encountered when relying solely on the VIIRS split window radiances for the k-d tree search. Over more uniform surfaces like the ocean, the split window provides a sense of total column moisture that is partially correlated with total column $\mathrm{SO}_{2}$. When the $\mathrm{SO}_{2}$ amounts are smaller, this becomes more difficult over land, where surface emissivity changes from one terrain to another are also influencing the k-d tree search.

\subsection{Tracking Volcanic $\mathrm{SO}_{2}$ over Time with AHI/TROPOMI Fusion}

To study the potential of tracking the $\mathrm{SO}_{2}$ plume over time, the TROPOMI $\mathrm{SO}_{2}$ measurements over Krakatoa, Indonesia, on December 23, 2018 are paired with the AHI band 10 (centered at $7.35 \mu \mathrm{m}$ with bandwidth of $0.2 \mu \mathrm{m}$ ) radiances in a spatial and then temporal fusion. Band 10, instead of the split window bands at 11.2 (bandwidth 0.8) $\mu \mathrm{m}$ and 12.4 (bandwidth 1.0) $\mu \mathrm{m}$ (AHI bands 14 and 15), is chosen to emphasize the mid-troposphere and reduce the influence of the Earth surface in the $\mathrm{k}$-d tree search. Band 10 has sensitivity to $\mathrm{SO}_{2}{ }^{12}$ It shows $20 \%$ absorption for an $\mathrm{SO}_{2}$ cloud of 100 DU. At time $t_{0}$, a k-d tree search on radiance and geolocation data is performed using AHI band 10 to enable the spatial fusion of TROPOMI $\mathrm{SO}_{2}$ measurements (in DU). This establishes a TROPOMI HIRES depiction of $\mathrm{SO}_{2}$ (i.e., $7 \mathrm{~km}$ is transferred to $2 \mathrm{~km}$ resolution) at $t_{0}$.

The TROPOMI $\mathrm{SO}_{2}$ product of the Krakatoa eruption on December 23 and 24, 2018 is shown in Figs. 4(a) and (c). The associated AHI/TROPOMI fusion results are shown in

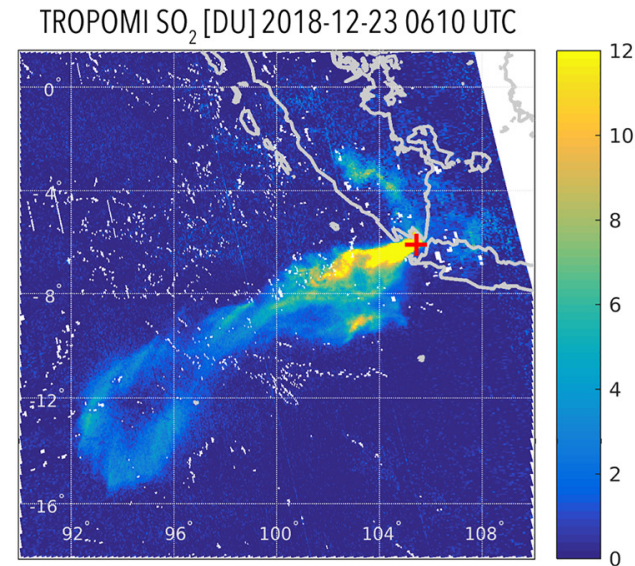

(a)

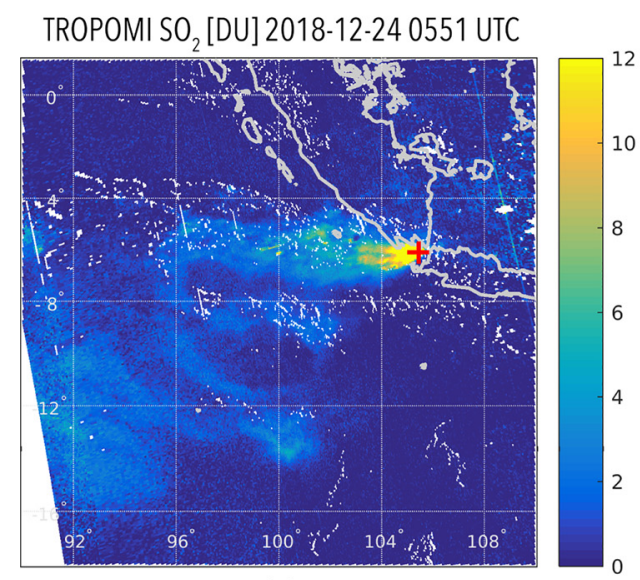

(c)

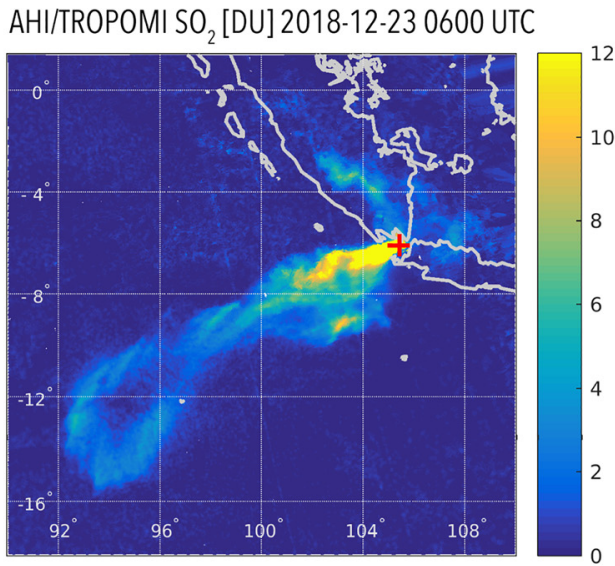

(b)

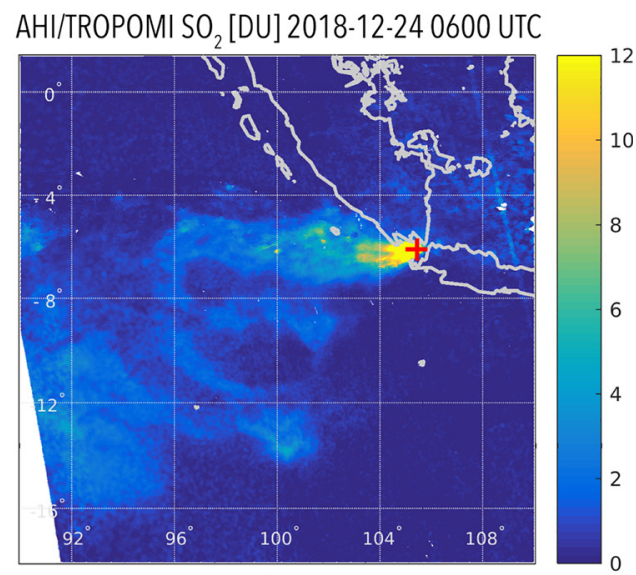

(d)

Fig. 4 (a) TROPOMI SO 2 [DU] emitted from Krakatoa on December 23, 2018 at 0610 UTC; (b) AHI/TROPOMI $\mathrm{SO}_{2}$ spatial fusion results on December 23, 2018 at 0600 UTC; and (c), (d) same scenario but $24 \mathrm{~h}$ later (on December 24, 2018, around 0600 UTC). 


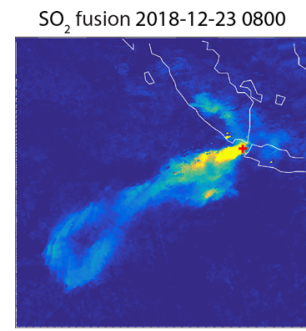

$\mathrm{SO}_{2}$ fusion $2018-12-232000$

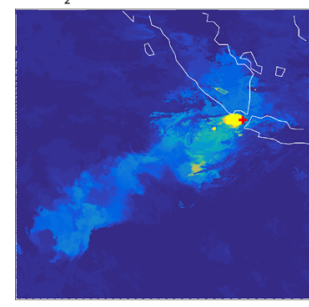

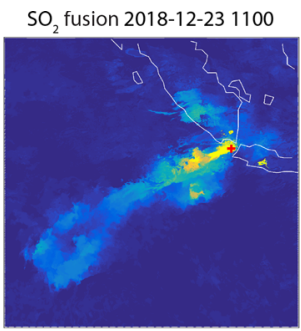

$\mathrm{SO}_{2}$ fusion 2018-12-23 2300

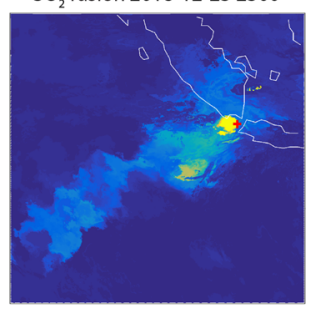

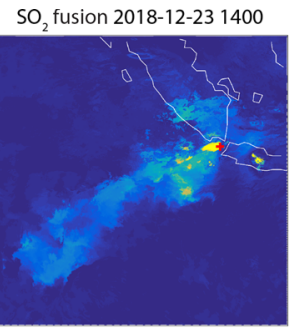

$\mathrm{SO}_{2}$ fusion 2018-12-24 0300

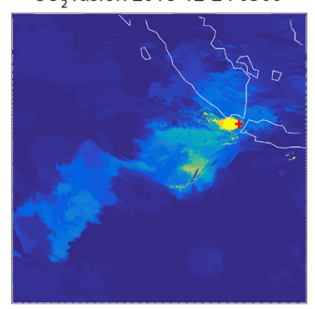

SO fusion 2018-12-23 1700

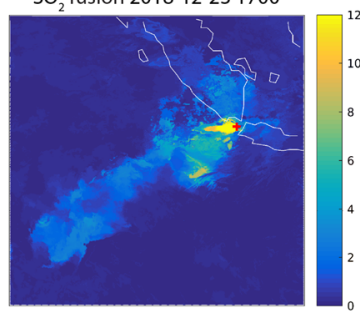

SO fusion 2018-12-24 0600

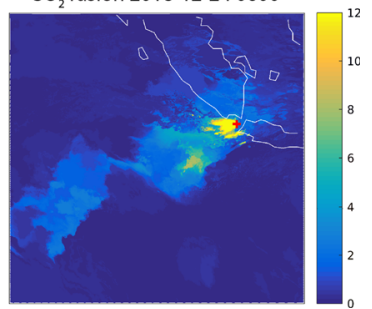

Fig. $5 \mathrm{AHI} / \mathrm{TROPOMI} \mathrm{SO}$ [DU] temporal fusion at 0800, 1100, 1400, 1700, 2000, and 2300 UTC on December 23 and 0300 and 0600 UTC on December 24, 2018 (for the Krakatoa, Indonesia, volcanic eruption).

Figs. 4(b) and (d). The latter, created via spatial fusion (with $N=5$ ) at times close to the TROPOMI measurement times, offer dramatic improvement in depiction of the spatial detail associated with the $\mathrm{SO}_{2}$ plume as well as filling data gaps.

As described in Sec. 2.2, at subsequent times, a k-d tree search is engaged between AHI band 10 radiances at the current $t_{i}$ and previous $t_{i-1}$ to select the five best matches and then the associated TROPOMI HIRES products (from the previous time step $t_{i-1}$ ) are averaged. This creates a new TROPOMI HIRES product (i.e., $\mathrm{SO}_{2}$ depiction at time $t_{i}$ ). Figure 5 shows a selection of hourly AHI/TROPOMI fusion depictions over a 24-h period, namely every $3 \mathrm{~h}$ starting at 800 UTC on December 23.

Although the gradual change in the $\mathrm{SO}_{2}$ outflow shows up in the temporal fusion products, it is noted that the extension of the AHI/TROPOMI temporal fusion $24 \mathrm{~h}$ onward from $0600 \mathrm{UTC}$ on December 23 generates a distribution of $\mathrm{SO}_{2}$ that is somewhat different from that shown in the AHI/TROPOMI spatial fusion at 0600 UTC on December 24 [see Fig. 4(d)]. However, despite missing the details of the actual $\mathrm{SO}_{2}$ distribution, the 24-h process indicates the subtle switch to northwesterly flow.

This example clearly indicates that there is a limit to how long temporal fusion can be sustained. The question is how far in space and time can the spatial/temporal fusion be used? The answer varies with mesoscale/synoptic situations. A validation study of ABI/CrIS temperature and moisture profile fusion ${ }^{8}$ found that fusion results compared with radiosonde observations (RAOB) on 30 different occasions were within the expected variability of RAOB/retrieval comparisons for at least three hours. In this Krakatoa example, for the times near the colocation time, temporal fusion offers an early indication of likely flow.

\subsection{Delineating $\mathrm{NH}_{3}$ with VIIRS/CrIS Fusion of MUSES Trace Gas Retrievals}

To improve the depiction of ammonia $\left(\mathrm{NH}_{3}\right)$ associated with fires in California on August 6, 2018, VIIRS split window data are used in a k-d tree search to fuse $\mathrm{CrIS} \mathrm{NH}_{3}$ profiles at $14 \mathrm{~km}$ to $750 \mathrm{~m}$ pixel resolution. As mentioned above and described in Ref. 2, the product fusion approach contains two main steps: first, a k-d tree search is done on VIIRS split window (bands 15 and 16 at 10.8 and $12.0 \mu \mathrm{m}$, respectively) pixel (HIRES) and FOV (LORES) spatial resolution radiance and associated geolocation data. Here $N=2$, i.e., the two FOVs that best match each of the imager pixels are retained. The change to $N=2$ was made to emphasize the best matches in a localized search near the fire. The split window search is not optimum for $\mathrm{NH}_{3}$ profile fusion but it works reasonably well when the surface terrain is similar. Fires produce a drastic change in nearby terrain and hence matching similar terrains is very important and more likely 


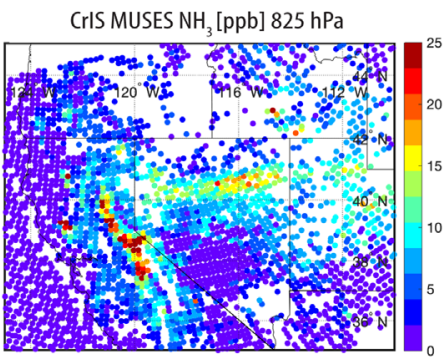

VIIRS/CrIS fusion MUSES NH 3 [ppb] $825 \mathrm{hPa}$

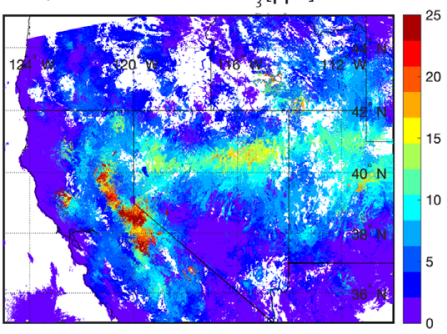

CrIS MUSES NH ${ }_{3}[\mathrm{ppb}] 909 \mathrm{hPa}$

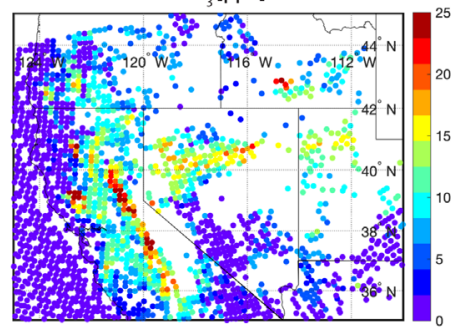

(a)

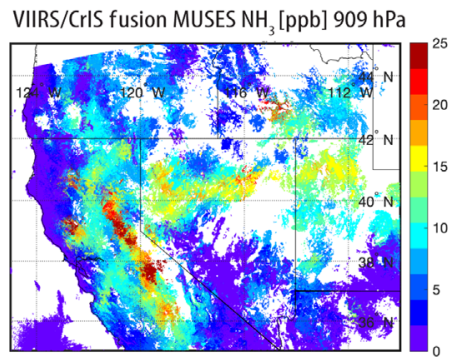

(b)

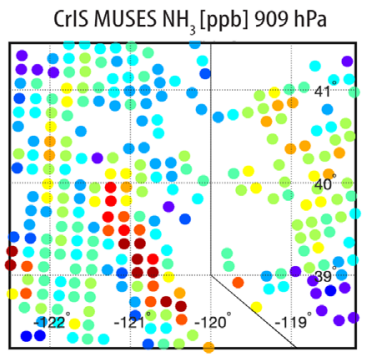

VIIRS/CrIS fusion MUSES NH $\mathrm{H}_{3}[\mathrm{ppb}] 909 \mathrm{hPa}$

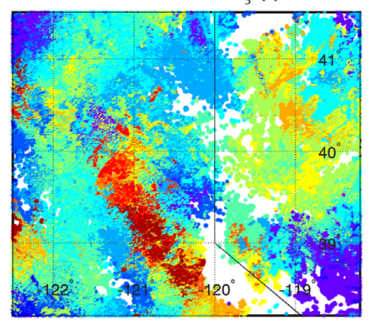

Fig. 6 (a) CrIS MUSES NH$H_{3}$ retrievals at $825 \mathrm{hPa}$ (left), at $909 \mathrm{hPa}$ (middle), and at $909 \mathrm{hPa}$ for an enlarged area (right). (b) The corresponding VIIRS/CrIS fusion of MUSES $\mathrm{NH}_{3}$ retrievals.

with $N=2$ than $N=5$. Then the MUlti-SpEctral, MUlti-SpEcies, MUlti-SEnsors (MUSES) ${ }^{13}$ trace gas retrievals are averaged over the two best LORES matches to provide a HIRES trace gas product. If the trace gas product contains missing values (e.g., due to clouds), the respective FOVs are excluded from the averaging step. Here the operational VIIRS cloud mask is used. Also the MUSES quality flag suggests low confidence in some retrievals and creates additional empty spaces. $\mathrm{NH}_{3}$ fields are shown at two pressure levels ( 825 and $909 \mathrm{hPa}$ ) in Fig. 6 but fusion products can be computed at any level (or any pressure range in the case of column amounts). The peak $\mathrm{NH}_{3}$ concentrations are similar for the two levels but the areal spread of the fusion product is greater lower in the troposphere.

Specific objectives of further research using MUSES data are to evaluate if the fine-scale structure of the fusion product is real (e.g., through comparison with data from aircraft instruments during air quality field campaigns), and if fusion is able to provide important information on, e.g., wildfire detection and plume characteristics to benefit operational fire management.

\subsection{Tracking CO Emissions from Industrial, Vehicle, and Fire Sources with AHI/TROPOMI Fusion}

To track $\mathrm{CO}$ emission and transport, $\mathrm{AHI}$ and TROPOMI data are combined to create a fusion CO product. To perform the k-d tree search, AHI channels $13(10.3 \mu \mathrm{m})$ and $15(12.4 \mu \mathrm{m})$ are used in an attempt to represent the total column of CO. Figure 7(a) shows TROPOMI CO concentration ${ }^{14}$ over northeastern China on March 23, 2020 at $\sim 0440$ UTC, which corresponds to 12:40 p.m. local time or China standard time (CST). Two geographical regions in northeastern China are investigated: first, the Hebei Province, a highly developed manufacturing (e.g., iron and steel) and transportation center, which contains some of the most polluted Chinese cities (see e.g., Ref. 15 for real-time air quality maps) such as Handan and Xingtai (south of Beijing), and second, the less prosperous region surrounding the city of Baicheng in the northwestern part of the Jilin Province, which is dominated by agriculture and forestry industry.

AHI/TROPOMI CO fusion is carried out over $16 \mathrm{~h}$ in 30-min time intervals, starting at 22 UTC on March 22 and ending at 14 UTC on March 23 (i.e., going both forward and backward in time with respect to the TROPOMI overpass). This time period corresponds to 6 through 22 CST on March 23, 2020. Fusion results at selected time steps are shown in Figs. 7(b)-(f). 


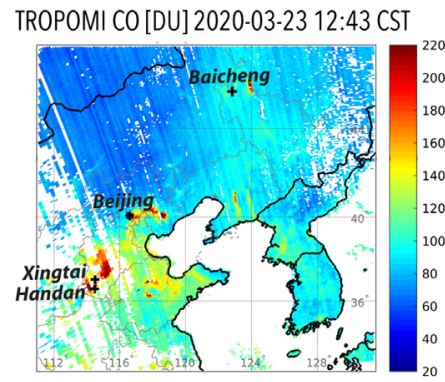

(a)

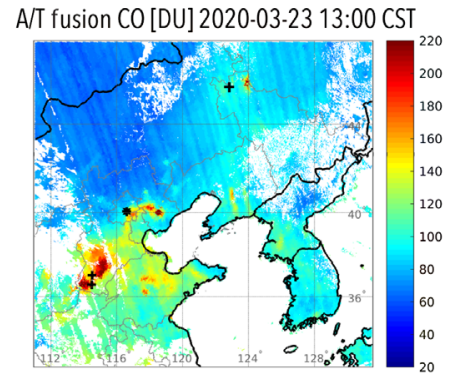

(d)

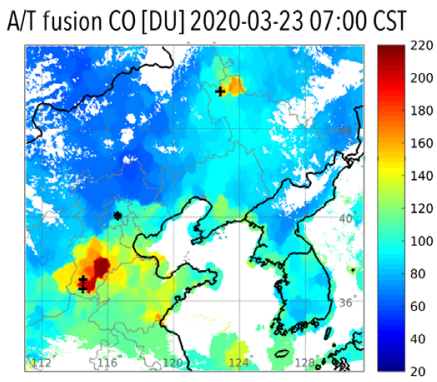

(b)

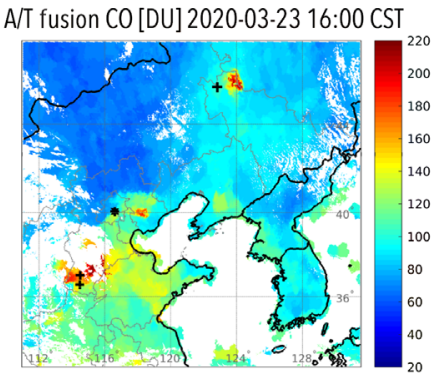

(e)

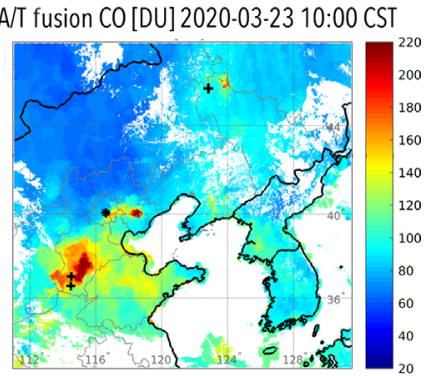

(c)

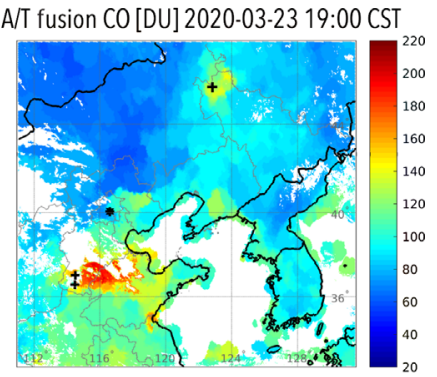

(f)

Fig. 7 (a) TROPOMI CO [DU] over NE China on March 23, 2020 at 12:43 CST or 0443 UTC. (b)-(f) AHI/TROPOMI fusion results at 7, 10, 13, 16, and 19 CST on March 23, 2020. Selected cities (Beijing, Baicheng, Handan, Xingtai) are marked and labeled in (a).

The increase in detail accomplished through spatial fusion for the overpass time and also the progression from antecedent to subsequent times by temporal fusion yields interesting new information. Figure 7(d) shows the spatial fusion product at a time closest to the TROPOMI measurement time [given in Fig. 7(a)]. The fusion detects very similar CO features but at higher spatial resolution. The changes in $\mathrm{CO}$ (in terms of amount and location) at each successive time step is dictated in the temporal fusion process by the AHI radiances where the k-d tree searches for the five best matching pixel radiances in the previous time to a given pixel radiance in the new time (and then the average of the associated five previous AHI/TROPOMI CO amounts becomes the new AHI/TROPOMI temporal fusion CO estimate). Strong and steady CO emissions are evident in the areas associated with industrial, economic, and social developments concentrated near the cities of Xingtai and Handan. The region near Baicheng in the north shows overall slightly lower but at the same time more fluctuating $\mathrm{CO}$ amounts, especially about $70 \mathrm{~km}$ east of the city of Baicheng along the Jilin and Heilongjiang Province border. For example, high CO concentrations occur at 10:00 CST (0200 UTC) and 16:00 CST (0800 UTC) as can be seen in Figs. 7(c) and (e), respectively. As will be discussed later, these $\mathrm{CO}$ emissions in northern Jilin are likely due to sporadic biomass burning events.

The 16-h AHI/TROPOMI fusion run can also be displayed as time sequences of $\mathrm{CO}$ concentrations at the selected locations to aid the study the diurnal variations in urban/industrial pollution. Figure 8 describes the temporal change in $\mathrm{CO}$ at the locations of Handan and Xingtai, two rapidly growing industrial cities in the southwest part of the Hebei Province. Both cities rank near the top of China's most polluted cities.

Figures 8(a) and (b) show the AHI/TROPOMI fusion results in the upper panels and hourly ground observations of $\mathrm{CO}$ (from Ref. 16) in the lower panels. The ground data are from the China Air Quality Online Monitoring and Analysis Platform (AQOMAP) that is operated in China as a public welfare software platform. It currently offers vast weather information data hourly from 367 cities nationwide, including the air quality index, particulate matter (such as PM2.5 and PM10), $\mathrm{SO}_{2}$, nitrogen dioxide, ozone, $\mathrm{CO}$, temperature, humidity, wind level, wind direction, satellite cloud image, and other monitoring items. The platform provides historical data query and analysis since December 2013 and includes real-time monitoring, monitoring 

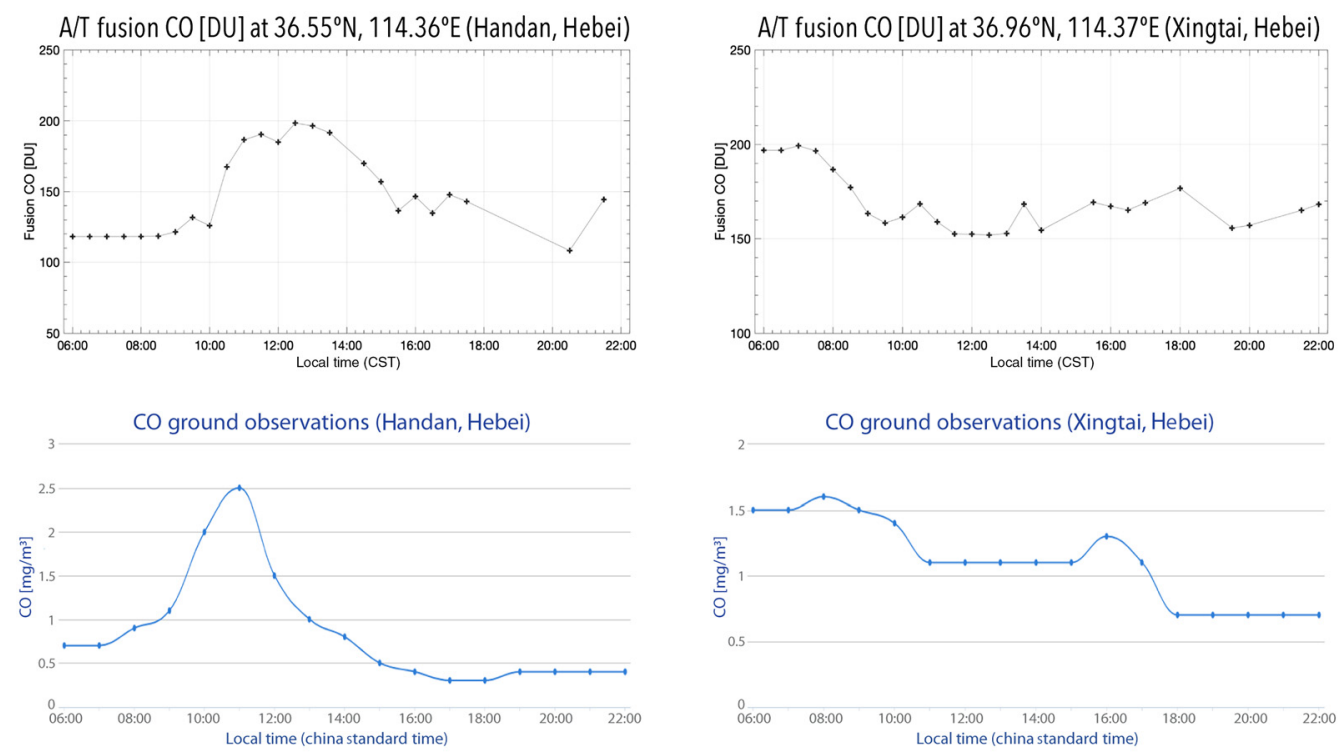

(a)

(b)

Fig. $8 \mathrm{AHI} / \mathrm{TROPOMI} \mathrm{CO}$ fusion time series (top) and AQOMAP ground observations (bottom) for the cities of (a) Handan and (b) Xingtai in the Hebei Province. The time period shown is 06:00 until 22:00 CST on March 23, 2020, which is 2200 UTC on March 22, 2020 until 1400 UTC on March 23, 2020.

curves, period statistics, city analysis, city distribution, province distribution, global distribution, satellite cloud map, city comparison, statistical ranking, historical analysis, and correlation mining and other functions.

According to Fig. 8(a), ground detection of $\mathrm{CO}$ emissions in Handan rises steadily during the day reaching a maximum $\left(\sim 2.5 \mathrm{mg} / \mathrm{m}^{3}\right)$ near the middle of the day (and close to the TROPOMI time) before the concentrations decrease in the local evening hours. Constant low values last throughout the night. This signature can be attributed to daily industrial activity, as well as heavy air and road traffic between the major cities in the Hebei Province. The satellite fusion estimates show a strong peak of $\sim 200 \mathrm{DU}$ arising at 11:00 CST but sustained twice as long (4 $\mathrm{h}$ in the satellite fusion versus $2 \mathrm{~h}$ from the ground). Carbon monoxide emissions from Xingtai are displayed in Fig. 8(b). A gradual morning drop in $\mathrm{CO}$ detected in the ground observation (1.5 to $1 \mathrm{mg} / \mathrm{m}^{3}$ ) is found in the satellite fusion estimates (200 to $\left.150 \mathrm{DU}\right)$ as is the afternoon rise, although the satellite fusion is more gradual and occurs roughly $2 \mathrm{~h}$ later.

These ground and satellite comparisons will necessarily differ. Ground measurements are made at the source of the emission, whereas satellite determinations are time interpolated area averages over several kilometers of the total column of the emission in the troposphere, which often lingers well after the emission stops. Nonetheless, one can conclude that they are marking the hourly changes in CO concentration for these two cities on March 23, 2020 with similar results.

Figure 9 focuses on the region surrounding Baicheng in the northern Jilin Province (shown near the top of Fig. 7). Figure 9(a) shows TROPOMI CO concentration as in Fig. 7(a) but for the zoomed-in area. Two locations $\sim 70 \mathrm{~km}$ east of Baicheng, where TROPOMI found high CO concentrations, are marked as "1" and "2" in Fig. 9(a). These high CO emissions are produced by fires (likely human-induced biomass burning), which is confirmed by high VIIRS M13 $(4.05 \mu \mathrm{m})$ brightness temperatures (BTs) visible as darkest spots (and highlighted in red) in Fig. 9(e).

The city of Baicheng and the two locations near the fires are marked as "+" in Figs. 9(b)-9(d) and 9(f)-9(h), displaying AHI band $2(0.51 \mu \mathrm{m})$ and band $7(3.85 \mu \mathrm{m})$ measurements and the CO fusion results at 13:00 CST (0500 UTC) and 16:00 CST (0800 UTC). The visible $0.51 \mu \mathrm{m}$ (or "green") band provides daytime observations related to land, clouds, and aerosols and is useful for air pollution studies, whereas the shortwave IR band centered at $3.85 \mu \mathrm{m}$, which 


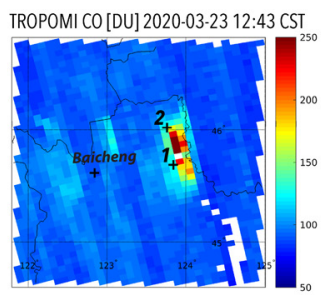

(a)

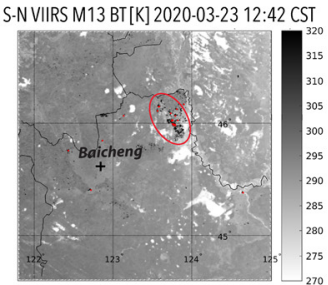

(e)

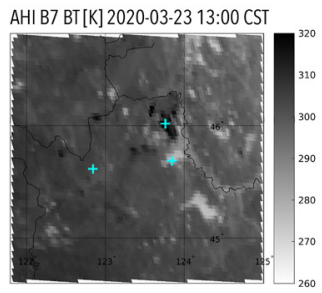

(b)

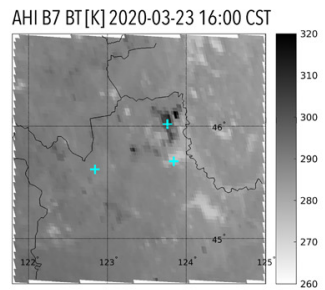

(f)

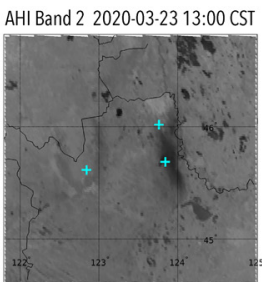

(c)

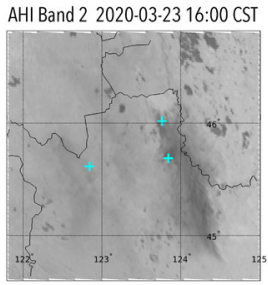

(g)

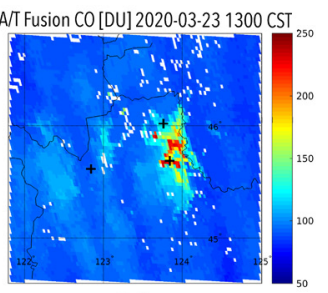

(d)

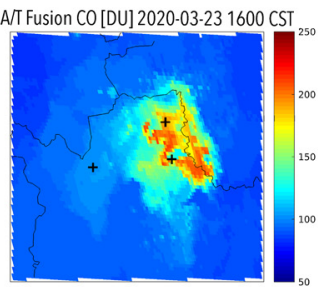

(h)

Fig. 9 (a) TROPOMI CO [DU] near Baicheng (northwest Jilin Province) on March 23, 2020 at 12:43 CST or 0443 UTC; (b) AHI band $7(3.85 \mu \mathrm{m})$ BTs at $13 \mathrm{CST}$; (c) AHI band $2(0.51 \mu \mathrm{m})$ radiances at $13 \mathrm{CST}$; (d) AHI/TROPOMI CO fusion results at $13 \mathrm{CST}$; (e) S-NPP VIIRS $4 \mu \mathrm{m}$ band BTs at $13 \mathrm{UTC}$; (f) AHI band $7(3.85 \mu \mathrm{m})$ BTs at $16 \mathrm{CST}$; $(\mathrm{g}) \mathrm{AHI}$ band $2(0.51 \mu \mathrm{m})$ radiances at $16 \mathrm{CST}$; and (h) AHI/TROPOMI CO fusion results at $16 \mathrm{CST}$.

senses both emitted terrestrial and reflected solar radiation, can be used to identify fire hot spots. The fires are evident in Figs. 9(b) and 9(f), with the hottest ones found near location 2 (northernmost "+" marker). Temperatures of the surrounding surface area decrease from 1 to $4 \mathrm{pm}$ local time while the temperatures of the hot spots remain high. Figs. 9(c) and 9(g) of AHI band 2 radiances clearly show dark areas of smoke plumes growing and blowing south during the $3 \mathrm{~h}$. The smoke plume in Fig. 9(g) and the CO plume in Fig. 9(h) extend roughly the same distance south but the stronger concentration of $\mathrm{CO}$ remains closer to the fire.

The $\mathrm{CO}$ ground and satellite 16-h time sequence comparison for Baicheng is shown in Fig. 10(a). The timing of the relatively strong emissions from Baicheng found in the ground and satellite determinations is within $1 \mathrm{~h}$ for both in the morning and the afternoon. The magnitude of the peak emissions is reversed. It is greater in the morning for AHI/TROPOMI fusion but greater in the afternoon for the AQOMAP. Overall, the Baicheng comparison shows similar changes in time. An early CO peak at 7 CST is followed by a decrease to a minimum at 13 CST, which then rises back up to another peak at 18 (20) CST in the fusion estimates (ground observations). The ground observed low-level $\mathrm{CO}\left(\sim 1 \mathrm{mg} / \mathrm{m}^{3}\right)$ is tracked nicely by the satellite fusion product $(\sim 150 \mathrm{DU})$.

Figure 10(b) illustrates the situation near the fires $\sim 70 \mathrm{~km}$ east of Baicheng. The AHI/ TROPOMI fusion timeline, shown in the upper panel, is from the location marked as "1" in Fig. 9(a). Because no ground data are available to validate the fusion results, a time sequence of AHI band $7(3.85 \mu \mathrm{m})$ BTs upwind at the fire source at location " 2 " (70 km north where the hottest temperatures are found) is shown as a correlative measurement (see for Fig. 9 discussion above) in the lower panel. The shapes of the ground and satellite time sequences shown in Fig. 10(b) are very similar, with the largest $\mathrm{CO}$ emissions being observed at the location downwind, around midday, about $1.5 \mathrm{~h}$ after the temperatures associated with the primary source fires reach a maximum.

These comparisons of time sequences of $\mathrm{CO}$ detection from the AHI/TROPOMI fusion and ground monitors offer credence that temporal fusion can reveal the growth and dissipation of $\mathrm{CO}$ presence in the atmosphere at a given location for several hours before and after TROPOMI overpass $(8 \mathrm{~h}$ before and after are shown in these three cases for Handan, Xingtai, and Baicheng). The ground temperatures of agricultural burning near Baicheng and the associated increase in downwind $\mathrm{CO}$ concentrations are also well depicted in spatial and temporal AHI/ TROPOMI fusion. 

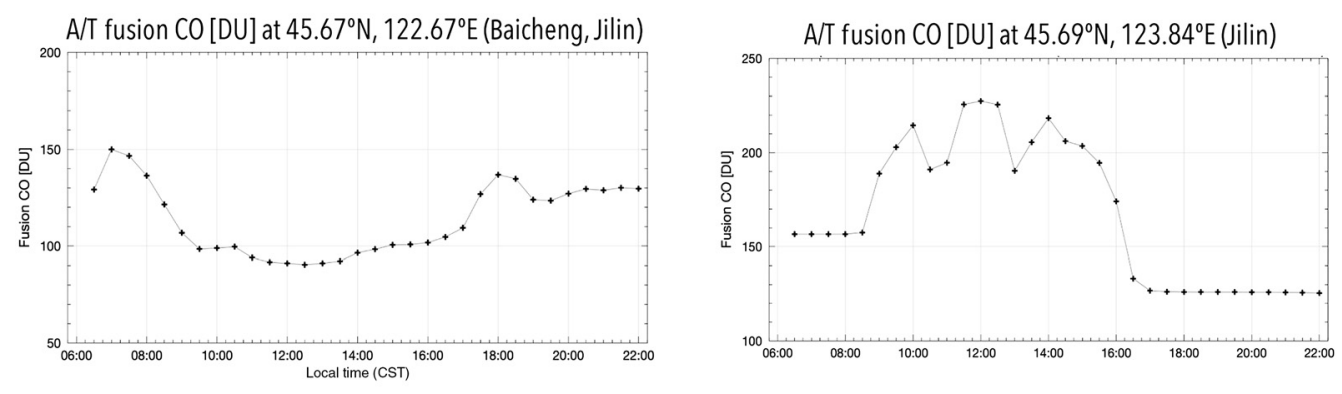

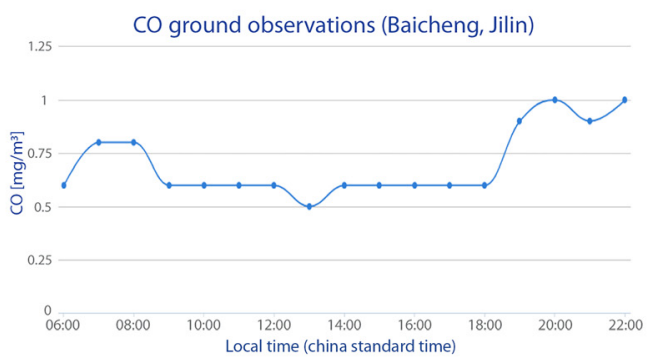

(a)

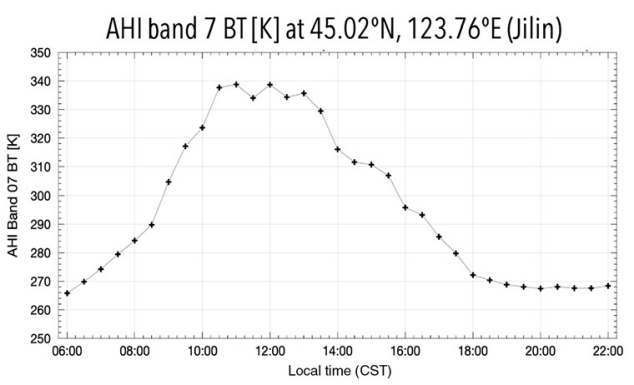

(b)

Fig. 10 (a) Time series of AHI/TROPOMI CO fusion (top) and AQOMAP ground observations (bottom) for Baicheng (Jilin Province). (b) Time series of AHI/TROPOMI CO fusion (top) and AHI band $7(3.85 \mu \mathrm{m})$ BTs (bottom) for locations $\sim 70 \mathrm{~km}$ east of Baicheng. The time period shown is 06:00 until 22:00 CST on March 23, 2020, which is 2200 UTC on March 22, 2020 until 1400 UTC on March 23, 2020.

\section{Summary and Conclusions}

This paper presents trace gas product fusion with imager measurements, where the results of a $\mathrm{k}-\mathrm{d}$ tree search of imager radiances at pixel and FOV resolution is directly applied to collocated trace gas concentrations. For each imager pixel, the trace gas measurements associated with selected FOVs (found through the imager-only k-d tree search) are averaged. This results directly in a trace gas product fusion that offers more spatial detail in the distribution of $\mathrm{CO}, \mathrm{SO}_{2}$, and $\mathrm{NH}_{3}$. The high frequency of AHI measurements is also utilized in the AHI/TROPOMI fusion results when the $\mathrm{k}-\mathrm{d}$ tree search is applied to each set of AHI radiances in time sequence and connected to the AHI/TROPOMI fusion product from the adjacent time period. Thus trace gas products are fused into high spatial as well as high temporal resolution. Overall, the case studies presented here (that include volcanic $\mathrm{SO}_{2}$ emissions from the March 2018 Popocatépetl and December 2019 Krakatoa eruptions, $\mathrm{NH}_{3}$ dissemination from California fires, and diurnal changes in $\mathrm{CO}$ associated with urban/industrial pollution and rural agricultural burning in China) suggest the possibility of inferring information from trace gas level 2 products at imager high spatial and temporal resolution. Although correlative ground comparisons offer encouragement regarding the viability of the trace gas fusion products, error bounds have not been addressed. Future planned work includes the pursuit of several case studies during field experiments with additional verification from the ground and aircraft.

The combination of vertical profile, high spatial, and better than hourly resolutions for monitoring tropospheric air quality as well as convective instability will be realized in the near future. The Geosynchronous Interferometric Infrared Sounder along with an advanced imager have been onboard Fengyun- 4 series. ${ }^{17}$ Geostationary high spectral resolution infrared sensors along with geostationary trace gas instruments are also planned for the first half of the 2020s. The GEO imager and LEO sounder (or trace gas) fusion offers an early look at the remote sensing capabilities that will become possible.

This fusion algorithm combining vertical resolution of hyperspectral sounding products (or the trace gas concentrations determined with a TROPOMI-like instrument) and horizontal plus temporal resolution of imager radiances is computationally efficient and stable. It can, therefore, 
be utilized in real-time operations of severe weather (or volcano, fire, and industrial emissions) monitoring, warning, and prediction systems. In the main, we anticipate that this new data source can help to improve our understanding of the impact of trace gas emissions on atmospheric chemistry, local air quality, as well as global climate.

\section{Acknowledgments}

The authors gratefully acknowledge the support from NOAA (Grant No. NA20NES4320003) and the encouragement of Dr. Dan Lindsey (NOAA GOES Program Scientist). VIIRS, CrIS, AHI, and ABI Level 1 data have been provided courtesy of the Space Science and Engineering Center/University of Wisconsin-Madison. We acknowledge the usage of TROPOMI data from the ESA Copernicus Sentinel-5P Open Access Data Hub. The authors are grateful to Dr. Vivienne Payne (NASA JPL) and Ms. Karen Cady-Pereira (AER) for providing the MUSES data and for their interest of the trace gas fusion approach. We also thank our colleague Dr. Zhenglong Li (SSEC) for informative discussions about Chinese cities and available ground truth measurements. The authors declare no conflicts of interest.

\section{References}

1. E. Weisz, B. A. Baum, and W. P. Menzel, "Fusion of satellite-based imager and sounder data to construct supplementary high spatial resolution narrowband IR radiances," J. Appl. Remote Sens. 11(3), 036022 (2017).

2. E. Weisz and W. P. Menzel, "Imager and sounder data fusion to generate sounder retrieval products at an improved spatial and temporal resolution," J. Appl. Remote Sens. 13(3), 034506 (2019).

3. Y. Han et al., "Suomi NPP CrIS measurements, sensor data record algorithm, calibration and validation activities, and record data quality," J. Geophys. Res. Atmos. 118, 12734-12748 (2013).

4. W. P. Menzel et al., "Satellite based atmospheric IR sounder development and applications," Bull. Am. Meteorol. Soc. 99(3), 583-603 (2018).

5. D. Hillger et al., "First-light imagery from Suomi NPP VIIRS," Bull. Am. Meteorol. Soc. 94(7), 1019-1029 (2013).

6. J. P. Veefkind et al., "TROPOMI on the ESA Sentinel-5 precursor: a GMES mission for global observations of the atmospheric composition for climate air quality and ozone layer applications," Remote Sens. Environ. 120, 70-83 (2012).

7. K. Bessho et al., "An introduction to Himawari-8/9-Japan's new-generation geostationary meteorological satellites," J. Meteorol. Soc. Jpn. Ser. II 94(2), 151-183 (2016).

8. T. J. Schmit et al., "Introducing the next-generation advanced baseline imager on GOES-R," Bull. Am. Meteorol. Soc. 86(8), 1079-1096 (2005).

9. J. H. Friedman, J. L. Bentley, and R. A. Finkel, "An algorithm for finding best matched in logarithmic expected time," ACM Trans. Math. Softw. 3(3), 209-226 (1977).

10. J. Anheuser, E. Weisz, and W. P. Menzel, "Low earth orbit sounder retrieval products at geostationary earth orbit spatial and temporal scales," J. Appl. Remote Sens. 14(4), 048502 (2020).

11. N. Theys et al., "Sulfur dioxide retrievals from TROPOMI onboard Sentinel-5 precursor: algorithm theoretical basis," Atmos. Meas. Tech. 10, 119-153 (2017).

12. S. A. Ackerman et al., "Using the GOES Sounder to monitor upper level SO2 from volcanic eruptions," J. Geophys. Res. Atmos. 113, D14S11 (2008).

13. D. Fu et al., "High-resolution tropospheric carbon monoxide profiles retrieved from CrIS and TROPOMI," Atmos. Meas. Tech. 9, 2567-2579 (2016).

14. J. Landgraf et al., "Algorithm baseline document for sentinel-5 precursor: carbon monoxide total retrieval," SRON (Netherlands Institute for Space Research), document number: SRON-S5P-LEV2-RP-002, CI identification: CI-7430-ATBD, https://sentinel.esa.int/ documents/247904/2476257/Sentinel-5P-TROPOMI-ATBD-Carbon-Monoxide-TotalColumn-Retrieval. 
15. "The World Air Quality Project, Air Pollution in China: Real-Time Air Quality Index Visual Map," https://aqicn.org/map/china/.

16. "China Air Quality Online Monitoring and Analysis Platform," https://www.aqistudy.cn/.

17. J. Yang et al., "Introducing the new generation of Chinese geostationary weather satellites, Fengyun-4,” Bull. Am. Meteorol. Soc. 98(8), 1637-1658 (2017).

Elisabeth Weisz received her MS degree in theoretical physics and her doctorate in geophysics and meteorology from the University of Graz, Austria. She has been at the Space Science and Engineering Center (SSEC) of the University of Wisconsin-Madison since 2001, currently serving as a senior scientist. Her research focuses on the development of atmospheric sounding retrieval algorithm using satellite-based high-spectral resolution infrared radiance measurements.

W. Paul Menzel received his degree in physics from the University of Wisconsin-Madison (UW) in 1974. Thereafter, at the UW SSEC, he explored possibilities for sounding the Earth's atmosphere from satellites. In 1983, he joined NOAA to develop and test new remote sensing environmental products. Currently a senior scientist at SSEC, he studies cloud and water vapor trends. He is a fellow of the American Meteorological Society. 